\title{
It infects me, it infects me not: phenotypic switching in the fungal pathogen Cryptococcus neoformans
}

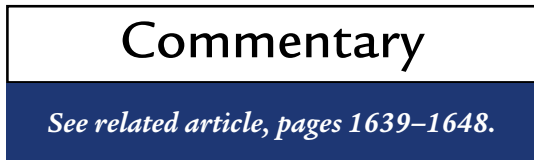

\author{
Cletus A. D'Souza and Joseph Heitman
}

Department of Genetics and the Howard Hughes Medical Institute, Duke University Medical Center, Durham, North Carolina, USA

Address correspondence to: Joseph Heitman, Howard Hughes Medical Institute, Departments of Genetics, Pharmacology and Cancer Biology, Microbiology, and Medicine, Duke University Medical Center, 322 CARL Building, Research Drive, Durham, North Carolina 27710 , USA. Phone: (919) 684-2824; Fax: (919) 684-5458; E-mail: heitm001@duke.edu.

J. Clin. Invest. 108:1577-1578 (2001). DOI:10.1172/JCI200114497.

Phenotypic switching has been observed in both prokaryotes and eukaryotes and involves stochastic switching between two or more alternative and heritable phenotypes, usually distinguished by surface antigenicity. This strategy, generally adopted by pathogens to escape recognition by the immune system and to adapt to a hostile host environment, results from spontaneous alterations in gene expression that arise at frequencies higher than standard spontaneous mutation rates. It differs from random spontaneous mutations that lead to phenotypic changes in individual cells in that it is reversible and readily detectable in a fraction of a cell population. Although phenotypic switching and filamentous dimorphic transitions in fungi are both reversible changes in gene expression that promote pathogenesis, phenotypic switching differs from fungal dimorphism in that the latter can occur in entire cell populations.

The basis and outcomes of phenotypic evolution of parasites and pathogens have been monitored in serial passage experiments that are frequently used in vaccine development (1). Such passage involves serial and horizontal transfer of parasites from one host to another to achieve experimental evolution; evolved traits are then compared with those of the progenitor parasite. In general, within-host competition between strains drives an increase in virulence of the parasites in a new host but an attenuation of virulence in the former host. In this issue of the JCI, Fries et al. show for the first time that the human fungal pathogen Cryptococcus neoformans can undergo phenotypic switching in vivo during passage in mice (2). Here, we present the current knowledge of phenotypic switching phenomena in this fungal pathogen.

C. neoformans is an encapsulated yeast that causes fatal meningitis in immunocompromised humans. The ability of this organism to cause chronic infections even after prolonged antifungal drug therapy may be in part attributable to phenotypic switching in this pathogen, rather than strictly a function of host immune function (3). Several lines of evidence had indicated that C. neoformans undergoes phenotypic switching in vitro and in vivo. First, relapse of cryptococcal meningitis results during persistent infection with a single infecting strain rather than reinfection with a new strain (4, 5). Second, serial Cryptococcus isolates from AIDS patients exhibit minor electrophoretic karyotype changes due to chromosome length polymorphisms, and they can differ in in vitro growth rates, capsule size, or virulence in mice (3). Additional changes that occur during chronic infection include stable alterations in cell membrane sterol composition and differences in the glucuronoxylomannan (GXM) structure of the capsule (6). Third, analysis of a standard strain maintained in various laboratories reveals significant differences in capsule size, melanin production, growth rates, and virulence in mice (7). Fourth, reversible switching between various colony morphologies (smooth, wrinkled, and pseudohyphal) has been observed in three strains including two serotypes $(8,9)$. This colony-type switching is associated with changes in virulence and in host inflammatory and antibody responses in rats. Switching to colony types that elicit minimal inflammation has there- fore been proposed as a mechanism for persistent infection. The frequency of colony-type switching is as high as 1 in 1000 to 1 in 100,000 cells, and variant colony morphology has been associated with altered cellular packing in the colony and quantitative and qualitative differences in capsular polysaccharide.

A caveat not to be ignored is that the signs of switching may be subtle and need not always produce readily apparent colony phenotypes. To conclusively demonstrate in vivo phenotypic switching, Fries et al. used inoculum sizes that precluded in vitro switched variants and showed that switching from a smooth to a mucoid phenotype occurred in two mouse strains (2). Switching was also associated with the production of a different antiphagocytic capsular polysaccharide and consequently increased virulence.

The environmental signals and mechanisms of phenotypic switching in C. neoformans remain largely unknown. No significant differences have been found in antifungal drug susceptibility of serial or relapse isolates, suggesting that drug resistance is not the main cause of persistent infection (10). Although phenotypic switching results in multiple phenotypes, karyotypic variability cannot always be linked to specific colony morphologies (9). In other pathogens, phenotypic switching is mediated by a variety of mechanisms, including transposition of mobile sequences, silencing of gene expression, activation of mutator genes, and rearrangement of repetitive DNA elements (11). For one switching C. neoformans strain, no DNA rearrangements involving C. neoformans repetitive element-1 (CNRE-1) were detected, although the possibility of rearrange- 
ment of other more recently discovered repetitive sequences could not be excluded (12). Phenotypic switching in C. neoformans could involve expansion or contraction of simple repetitive DNA sequences, as is observed in bacteria (13-15), or could involve epigenetic phenomena such as silencing and altered chromatin structure, as reported for the mating-type cassettes in Saccharomyces cerevisiae (16).

The pathogenic yeast Candida albicans has a switching system similar to that observed in C. neoformans. Phenotypic switching produces colony types reflecting dramatic differences in cellular architecture rather than antigenicity. Additionally, colony variants differ in virulence traits such as adherence to epithelial cells, protease production, and susceptibility to neutrophil-mediated killing, as well as antifungal drug susceptibility (17). The pleiotropic effect of switching has been shown to be due to differential gene expression and appears more likely due to a change at a key regulatory site, rather than spontaneous, independent changes at unlinked loci. One such candidate is the SIR2 gene, because a homozygous sir $2 /$ sir 2 mutant strain exhibits dramatically increased colony switching and karyotype variability, suggesting that phenotypic switching is controlled by genes involved in silencing (18). Additional switch control genes identified include EFG1, encoding a transcription factor also required for filamentation (19), and the histone deacetylase genes $H D A 1$ and $R P D 3$ that play distinct roles in the suppression of switching and are in turn controlled transcriptionally by switching (20).

In conclusion, Cryptococcus researchers may find important clues to the mechanisms of phenotypic switching by using the information available from C. albicans as a guide. Unraveling the regulatory circuits involved in phenotypic switching in C. neoformans will require isolation and characterization of genes involved in this process. Although such studies may be hampered by the stochastic nature of phenotypic switching, a clear understanding of the mechanics of this process may be useful in devising effective strategies to control the blight of switching fungal pathogens.

1. Ebert, D. 1998. Experimental evolution of parasites. Science. 282:1432-1435.

2. Fries, B.C., Taborda, C.P., Serfass, E., and Casadevall, A. 2001. Phenotypic switching of Cryptococcus neoformans occurs in vivo and influences the out come of infection. J. Clin. Invest. 108:1639-1648.

3. Fries, B.C., and Casadevall, A. 1998. Serial isolates of Cryptococcus neoformans from patients with AIDS differ in virulence for mice. J. Infect. Dis. 178:1761-1766

4. Klepser, M.E., and Pfaller, M.A. 1998. Variation in electrophoretic karyotype and antifungal susceptibility of clinical isolates of Cryptococcus neoformans at a university-affiliated teaching hospital from 1987 to 1994. J. Clin. Microbiol. 36:3653-3656

5. Spitzer, E.D., Spitzer, S.G., Freundlich, L.F., and Casadevall, A. 1993. Persistence of initial infection in recurrent Cryptococcus neoformans meningitis. Lancet. 341:595-596.

6. San-Blas, G., et al. 2000. Fungal morphogenesis and virulence. Med. Mycol. 38(Suppl.):79-86.

7. Franzot, S.P., et al. 1998. Microevolution of a standard strain of Cryptococcus neoformans resulting in differences in virulence and other phenotypes. Infect. Immun. 66:89-97.

8. Goldman, D.L., Fries, B.C., Franzot, S.P., Montella, L., and Casadevall, A. 1998. Phenotypic switching in the human pathogenic fungus Cryptococcus neoformans is associated with changes in virulence and pulmonary inflammatory response in rodents. Proc. Natl. Acad. Sci. USA. 95:14967-14972.

9. Fries, B.C., Goldman, D.L., Cherniak, R., Ju, R. and Casadevall, A. 1999. Phenotypic switching in Cryptococcus neoformans results in changes in cellular morphology and glucuronoxylomannan structure. Infect. Immun. 67:6076-6083.

10. Brandt, M.E., et al. 1996. Molecular subtypes and antifungal susceptibilities of serial Cryptococcus neoformans isolates in human immunodeficiency virus-associated Cryptococcosis. Cryptococca Disease Active Surveillance Group. J. Infect. Dis. 174:812-820.

11. Deitsch, K.W., Moxon, E.R., and Wellems, T.E. 1997. Shared themes of antigenic variation and virulence in bacterial, protozoal, and fungal infections. Microbiol. Mol. Biol. Rev. 61:281-293.

12. Cruz, M.C., et al. 1999. Rapamycin anti fungal action is mediated via conserved complexes with FKBP12 and the TOR kinase homologs in Cryptococcus neoformans. Mol. Cell. Biol. 19:4101-4112

13. Moxon, E.R., Rainey, P.B., Nowak, M.A., and Lenski, R.E. 1994. Adaptive evolution of highly mutable loci in pathogenic bacteria. Curr. Biol. 4:24-33.

14. High, N.J., Deadman, M.E., and Moxon, E.R. 1993. The role of a repetitive DNA motif $\left(5^{\prime}\right.$-CAAT- $\left.3^{\prime}\right)$ in the variable expression of the Haemophilus influenzae lipopolysaccharide epitope alpha Gal(1-4)beta Gal. Mol. Microbiol. 9:1275-1282.

15. Jonsson, A.B., Nyberg, G., and Normark, S. 1991 Phase variation of gonococcal pili by frameshift mutation in pilC, a novel gene for pilus assembly. EMBO J. 10:477-488.

16. Haber, J.E. 1998. Mating-type gene switching in Saccharomyces cerevisiae. Annu. Rev. Genet. 32:561-599.

17. Soll, D.R. 1997. Gene regulation during high-frequency switching in Candida albicans. Microbiology. 143:279-288.

18. Perez-Martin, J., Uria, J.A., and Johnson, A.D 1999. Phenotypic switching in Candida albicans is controlled by a SIR2 gene. EMBO J. 18:2580-2592.

19. Sonneborn, A., Tebarth, B., and Ernst, J.F. 1999. Control of white-opaque phenotypic switching in Candida albicans by the Efg1p morphogenetic regulator. Infect. Immun. 67:4655-4660.

20. Srikantha, T., Tsal, L., Daniels, K., Klar, A.J.S., and Soll, D.R. 2001. The histone deacetylase genes $H D A 1$ and $R P D 3$ play distinct roles in regulation of high-frequency phenotypic switching in Candida albicans. J. Bacteriol. 183:4614-4625. 